\title{
Construction and Establishment of a New Environmental Chamber to Study Real-Time Cardiac Development
}

\author{
Gülay Orhan, ${ }^{1}$ Stephan Baron, ${ }^{2}$ Kambiz Norozi, ${ }^{1}$ Jörg Männer, ${ }^{3}$ Oliver Hornung, ${ }^{2}$ Holger Blume, ${ }^{2}$ \\ Judith Misske, ${ }^{1}$ Bodo Heimann, ${ }^{2}$ Armin Wessel, ${ }^{1}$ and T. Mesud Yelbuz, ${ }^{1, \star}$ \\ ${ }^{1}$ Department of Pediatric Cardiology and Intensive Care Medicine, Hannover Medical School, Carl-Neuberg-Str. 1, \\ 30625 Hannover, Germany \\ ${ }^{2}$ Hannover Center of Mechatronics, Leipniz University of Hannover, Appelstraße 11, 30167 Hannover, Germany \\ ${ }^{3}$ Department of Anatomy \& Embryology, Georg-August-University of Göttingen, Kreuzbergring 36, 37075 Göttingen, Germany
}

\begin{abstract}
Heart development, especially the critical phase of cardiac looping, is a complex and intricate process that has not yet been visualized "live" over long periods of time. We have constructed and established a new environmental incubator chamber that provides stable conditions for embryonic development with regard to temperature, humidity, and oxygen levels. We have integrated a video microscope in the chamber to visualize the developing heart in real time and present the first "live" recordings of a chick embryo in shell-less culture acquired over a period of 2 days. The time-lapse images we show depict a significant time window that covers the most critical and typical morphogenetic events during normal cardiac looping. Our system is of interest to researchers in the field of embryogenesis, as it can be adapted to a variety of animal models for organogenesis studies including heart and limb development.
\end{abstract}

Key words: heart development, imaging, cardiac looping

\section{INTRODUCTION}

In the past, various imaging techniques have been employed to visualize the intricate processes of cardiovascular development in two or three dimensions to achieve a better understanding of the underlying mechanisms for the genesis of congenital heart defects during embryonic development (Kolker et al., 2000; Yelbuz et al., 2002a, 2002b, 2002c, 2003b, 2004; Soufan et al., 2003; Efimov et al., 2004; Borg et al., 2005; Tutarel et al., 2005). However, in most of these studies cardiac development could not be continuously documented over long time periods because nonbeating embryonic hearts were used or in vivo studies were conducted with long time gaps between periods of image collection. In our previous attempts at in vivo visualization of cardiovascular development, frequent time intervals between images did not allow an accurate assessment of the developmental process because of methodological deficiencies resulting from unstable environmental conditions (Yelbuz et al., 2002a, 2002b; Tutarel et al., 2005).

The goal of this study was to overcome these limitations by constructing and establishing a new environmental chamber that would guarantee both stable environmental conditions for the developing embryo and "live visualization" and recording of heart development over long periods

Received July 28, 2006; accepted February 21, 2007.

${ }^{\star}$ Corresponding author. E-mail: Yelbuz.Mesud@mh-hannover.de of time by means of an integrated video microscope system. We present the first real-time images of heart development in chick embryos in shell-less culture (SC) during cardiac looping acquired in this chamber.

\section{Materials And Methods}

\section{Embryo Preparation}

Fertilized chicken eggs (Lohmann Tierzucht GmbH, Cuxhaven, Germany) were incubated at $37.5^{\circ} \mathrm{C}$ and $70 \%$ humidity in a forced-draft incubator. After $48 \mathrm{~h}$ of incubation the intact chick eggshell contents were transferred to sterilized hexagonal polystyrene weighing boats in a Petri dish with water (temperature $37^{\circ} \mathrm{C}$ ) and checked for correct staging according to Hamburger and Hamilton (1951; HH). A chick embryo in SC at $\mathrm{HH}$-stage 15 was placed in the environmental chamber. Before each recording the cover of the Petri dish was removed to allow best image quality and replaced after filming to prevent dehydration of the extra-embryonic membranes.

\section{Environmental Chamber}

To fulfill our experimental requirements, a prototype of an environmental chamber was built in 2004 in cooperation with engineers from the Hannover Center of Mechatronics (Hannover, Germany). Over a period of 2 years it was tested 
and enhanced to meet the high demands of artificial and stable culture conditions for embryonic development.

Its internal cell consists of a $400 \times 400 \times 400 \mathrm{~mm}^{3}$ large stainless steel chamber (Fig. 1) with an isolating Plexiglas door at the front. Development of the chick embryos is recorded by an integrated microscope camera system, as described below. To avoid stressing the camera system with high temperature and moisture it was encapsulated in a tube of plastic, as shown in Figure 1A,B. At its lower end the camera system is separated from the inside of the chamber by a special isolating glass (Schott AG, Fiberoptics, Mainz, Germany) with a very low light absorption for high quality imaging.

A Linux-driven Arm9-Socket Computer (SC9328, Synertronixx $\mathrm{GmbH}$, Hannover, Germany) handles the acquisition of sensor data from several positive temperature coefficient resistors (Fig. 1B, arrow f) as well as from a combined temperature and humidity sensor SHT75 (Fig. 1B, arrow d) (Sensirion AG, Steafa, Switzerland). This sensor is factory calibrated and provides a high accuracy $( \pm 1.8 \%$ $\left.\mathrm{RH}, \pm 0.3^{\circ} \mathrm{C}\right)$. In addition, an oxygen sensor was integrated into the system (Fig. 1B, arrow e).

With the measured sensor data, a control algorithm calculates the appropriate actuating variables to provide a stable temperature and humidity. All parameters are displayed continuously on an external panel of the control unit of the chamber. Additionally these data can be recorded internally and downloaded by an ethernet connection to a normal PC. This allows arbitrary long-term recording of environmental data inside the chamber.

The necessary components controlling the climate inside the chamber are assembled in a tube system that is attached to the inner chamber. The regulation of the climate is processed by an axial blower, a piezo mist generator, and a heating/cooling unit. The axial blower provides air circulation in the tube system. The moisture generator produces an aerosol that is absorbed by the passing air for humidity control. The cooling/heating aggregate consists of two $80 \mathrm{~W}$ thermoelectric modules that are bonded to a heat sink. This allows a very precise, continuous temperature control.

The stability of the system, in particular with regard to humidity and temperature stability and distribution, was continuously improved. Now the chamber allows visualization and recording embryonic development under stable environmental conditions over incubation periods up to 11 days (Fig. 2). An even heat distribution throughout the chamber was established by an additional fan in the back of the chamber. The temperature distribution was checked both by incubation experiments of chick embryos in ovo and in SC (Fig. 1B; Table 1) and visually documented by an infrared camera (ThermaCAM S40, FLIR Systems GmbH, Frankfurt am Main, Germany).

\section{Imaging in the New Environmental Chamber}

For imaging, a digital high-speed video camera (PULNIX TM-6710 CL, JAI PULNIX Inc., Sunnyvale, CA), an inte- grated lens (without ocular optics) connected directly to the camera via a C mount adaptor, and a PC-based zoom and focus control were integrated with the environmental chamber. The integrated lens yields a magnification of approximately $150 \times$. A stage micrometer (scribed glass standard) was filmed at the same focal plane as the embryo to allow exact calibration of the magnification factor. For correct placement of the embryo in SC inside the chamber during recording, a height-adjustable positioning stage (LJ750; Compact Lab Jack, Thorlabs GmbH, Karlsfeld, Germany) was used. The digital high-speed video camera records at a frame rate of 120 frames/s. This imaging system is connected to a high-performance computer (Windows XP, Pentium 4, $3.0 \mathrm{GHz}, 512 \mathrm{MB}$ RAM, $80 \mathrm{~GB}$ HHD) for image processing. Lighting during the filming process is provided by a fiber optic cold white light source (Dual Gooseneck A08520, Schott AG, Fiberoptics, Mainz, Germany) placed outside of and controlled by flexible dual head tubes inside the chamber lateral to the positioning stage and to the camera tube (Fig. 1A,B). To ensure stable placement of the embryo, we fixed the Petri dish with glue strips on the positioning stage. Before each recording the chamber door was opened for a brief period of less than $1 \mathrm{~min}$, the embryonic position in the imaging field was checked and adjusted manually, and the chamber door closed again. The embryo was visualized and recorded for $4 \mathrm{~s}$ ( $=500$ frames) every $60 \mathrm{~min}$ over $46 \mathrm{~h}$. The video images were stored, later further processed off-line by contrast enhancement, and edited using a new special software solution (HeartMetrics, Physimetrics, Inc., Roswell, GA, and Video Savant, IO Industries Inc., London, ON). Finally, a movie was created that shows two full heart cycles per recording over $46 \mathrm{~h}$. Image series from this time-lapse movie are displayed in Figure 3 (for details see legend of Fig. 3).

\section{Statistical Analysis}

The data presented in Table 1 were analyzed using Excel 2000 (Microsoft) and Prism (GraphPad Software, Inc.). Results are reported as mean $\pm S D$. Groups were assessed using the nonparametric Mann-Whitney test.

\section{Results}

The new environmental chamber works as an incubator (Fig. 1A,B) and provides stable conditions as a key prerequisite to visualize and record embryonic development over long periods of time as shown in Figure 2.

The images taken by an infrared thermal camera confirm that heat distribution is even throughout the chamber. To prove that local heat distribution is also even over time we incubated chick embryos both in the chamber and the forced-draft incubator whereby the embryos in the chamber were placed in the front, lateral, and back portions, respectively. After $55 \mathrm{~h}$ of incubation we transferred all embryos 

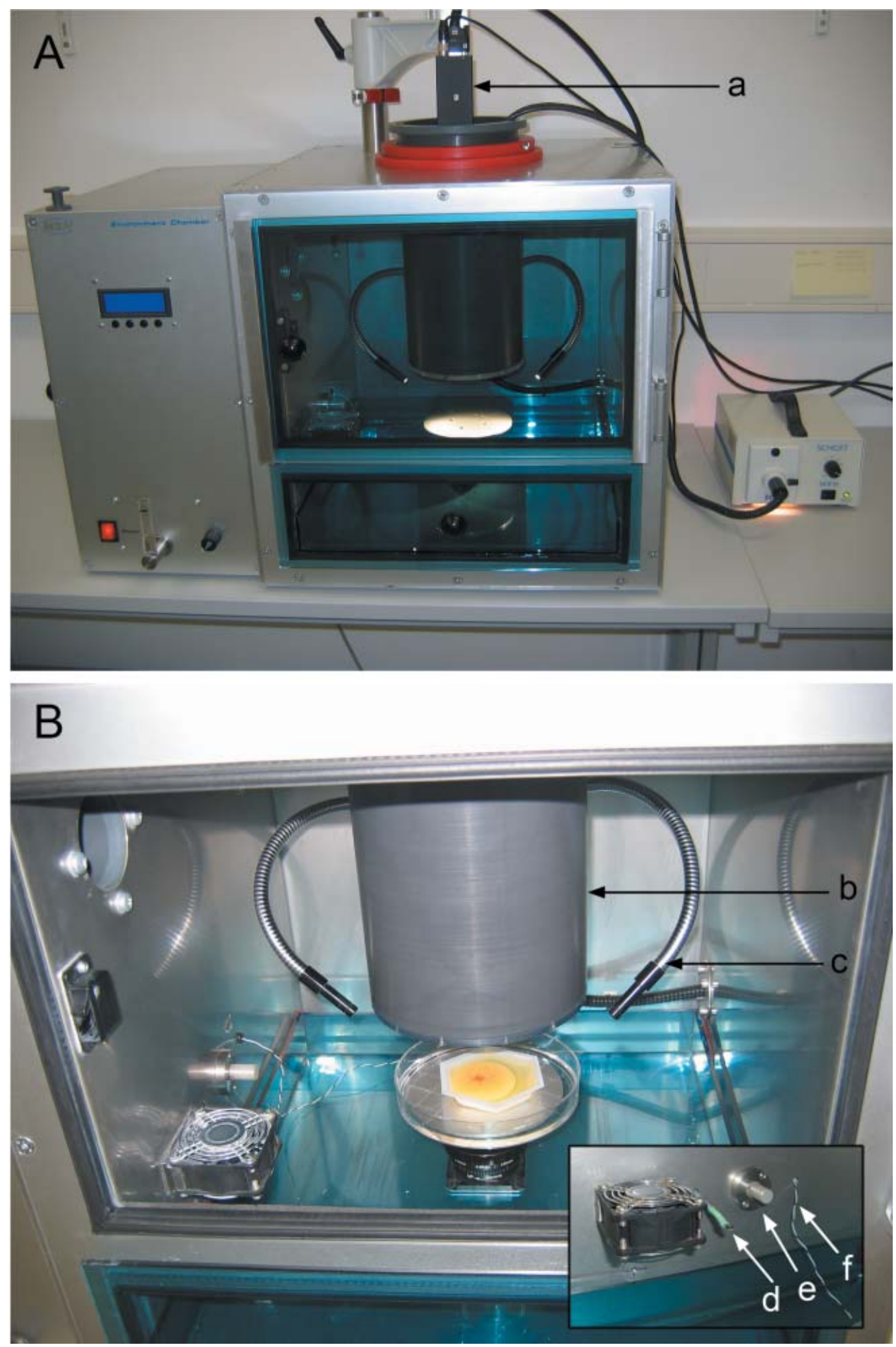

Figure 1. Environmental chamber with an integrated video microscope system. A: The chamber consists of a tube (arrow b in B) containing the video microscope system with a digital high-speed video camera (arrow a), positioning stage (below the tube), and a dual head cold white light on both sides of tube (arrow c in B) connected to the light source outside of the chamber. B: Close-up view of the chick embryo in SC on the positioning stage below the camera tube. Inserted image on the right shows the measurement unit within the chamber from a different angle with the combined temperature and humidity sensor (arrow d), oxygen sensor (arrow e), and one of the temperature coefficient resistors (arrow f). 


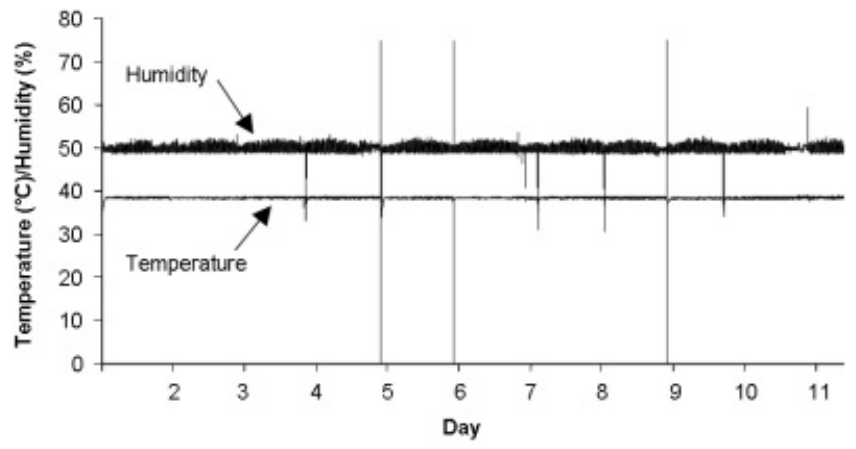

Figure 2. Environmental conditions in the chamber. The diagram depicts data that illustrate continuous and constant temperature $\left(38^{\circ} \mathrm{C}\right)$, humidity (50\%), (and oxygen; not shown) levels over a period of 11 days. The high vertical lines depicted in the graph for humidity and temperature represent software errors during recording of the data. Shorter lines reflect phases when the door of the chamber was opened briefly for control purposes.

Table 1. Development of Chick Embryos in Environmental Chamber versus Development in Forced-Draft Incubator ${ }^{a}$

\begin{tabular}{llr}
\hline & $\begin{array}{l}\text { Number of } \\
\text { embryos }\end{array}$ & $\begin{array}{r}\text { Stage according to HH } \\
(\text { mean } \pm S D) /\end{array}$ \\
Forced-draft & $\begin{array}{l}\text { Total: } n=20 \\
\text { incubator }\end{array}$ & $\begin{array}{l}\text { Fertilized and } \\
\text { developed: } n=18\end{array}$ \\
& $\begin{array}{l}\text { Total: } n=20 \\
\text { Environmentame }(\mathrm{h}) \text { ) } \\
\text { chamber }\end{array}$ & $\begin{array}{l}\text { Fertilized and } \\
\text { developed: } n=16\end{array}$ \\
& n.s. & $16.38 \pm 0.94 /(51-56 \mathrm{~h})$ \\
$p$-Value & & n.s.
\end{tabular}

${ }^{a}$ There are no significant differences in the development of embryos in the environmental chamber and the forced-draft incubator. n.s.: not significant.

into SC and staged according to HH. Compared to the embryos in the forced-draft incubator the embryos in the chamber reached equal stages irrespective of their positioning in the chamber (Table 1).

All the embryos incubated or incorporated into experiments developed normally and did not display any cardiac defects by external morphological analysis at different phases of development (T.M. Yelbuz, G. Orhan, J. Misske, S. Baron, \& K. Norozi, unpublished data).

Our time-lapse images, created from "live" recordings in the new environmental chamber with an integrated video microscope system demonstrate continuous visualization of the developing chick heart in SC during cardiac looping over an incubation period of 2 days (Fig. 3).

\section{Discussion}

In the present study we visualized for the first time the developing chick heart in SC in real time using a new environmental chamber with an integrated video microscope system (Fig. 1A,B). We were able to record and study cardiac development in living chick embryos over a period of $46 \mathrm{~h}$ under stable environmental conditions. This is in contrast to all previous imaging techniques (i.e., video light microscopy, ultrasound, confocal and scanning electron microscopy, high resolution MRI, and optical coherence tomography) that we and others have used for imaging of the developing embryonic heart before (Kolker et al., 2000; Yelbuz et al., 2002a, 2002b, 2002c, 2003b, 2004; Soufan et al., 2003; Efimov et al., 2004; Borg et al., 2005; Tutarel et al., 2005).

As cardiovascular physiology changes during embryonic development in a highly complex and carefully orchestrated manner, even minor negative factors or triggers could disrupt critical processes of heart development (De la Cruz \& Markwald, 1998; Keller, 1998; Harvey \& Rosenthal, 1999; Phoon, 2001; Tomanek \& Runyan, 2001; Burggren \& Warburton, 2005). The major limitation in previous attempts to visualize the beating developing heart over short and long periods was that the embryos had to be moved repetitively from an conventional incubator to a special stage and reincubated (Leatherbury et al., 1990; Tomita et al., 1991; Conway et al., 1997; Yelbuz et al., 2002a, 2002b; Li et al., 2003; Tutarel et al., 2005). This is true in studies of both chick and zebrafish embryos. Hereby measures such as a warm water bath, heat pellets, or lamps were used to maintain temperature stability (Leatherbury et al., 1990; Tomita et al., 1991; Conway et al., 1997; Yelbuz et al., 2002a, 2002b; Li et al., 2003; Tutarel et al., 2005). In our new environmental chamber the embryos could be kept in the same position in the chamber (Fig. 1B) during the whole recording process without any disturbances by change of temperature, humidity (Fig. 2), or oxygen levels (not shown).

The time-lapse images we show depict a significant time window that covers the most critical and typical morphogenetic events during normal cardiac looping (Fig. 3), as defined by Männer $(2000,2004)$. Because we know from previous (Yelbuz et al., 2002c, 2003a) and current studies (Ward et al., 2005) that alterations of cardiac looping can lead to various malformations of the heart, this and future studies could provide new insights in our understanding of normal and abnormal cardiac development.

Further, coupled with the emerging powerful technologies of molecular and cellular biology, new in vivo investigations in different experimental settings that result in heart defects could enable us to visually pinpoint the critical events and periods where, when, and how heart formation starts to go awry.

Finally, we think that our system, consisting of a chamber with an integrated video microscope system, is of inter- 

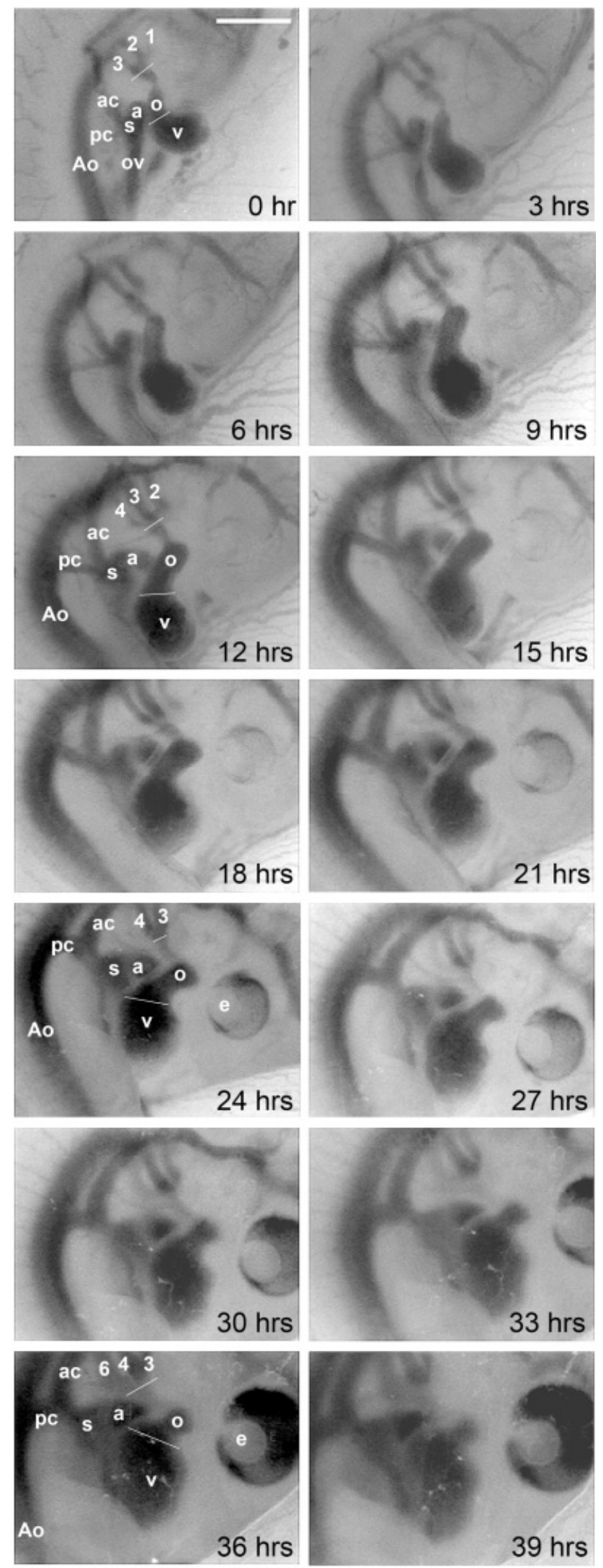

Figure 3. Image series from a time-lapse movie ("live" visualization). The images depict the developing heart in a chick embryo in SC during cardiac looping from the right lateral view in enddiastole recorded with a video microscope system integrated in the environmental chamber. The image series shown starts on incubation day 2 (HH-stage 15/16) and ends on incubation day 4 (HH-stage 22/23), which covers a period of $39 \mathrm{~h}$ depicted here in 3 -h intervals. The original time-lapse movie, not shown, covers a total of $46 \mathrm{~h}$ of continuous development in 1-h intervals up to day 4.5 (HH-stage 24). Note the change in length and morphology of the outflow tract (proximal and distal ends marked by white lines) and the significant growth of the primitive ventricles. 1,2,3,4,6: pharyngeal arch arteries; a, primitive atrium; ac, anterior cardinal vein; Ao, dorsal aorta; e, eye of the embryo; o, outflow tract; pc, posterior cardinal vein; s, sinus venosus; v, primitive ventricles; ov, omphalomesenteric vein; bar $=1 \mathrm{~mm}$. (The animation for this figure can be accessed at the Microscopy and Microanalysis website: www.journals.cambridge.org/jid_MAM. At the Orhan et al. article, please click on "Supplement Materials" to view the moving image.)

est to other researchers in the field, as it could be applied and adapted - with some limitations as described below-in a variety of animal models such as zebrafish, Xenopus, or mouse for particular studies of organogenesis, not limited to heart development only, such as limb development.

\section{Limitations}

This new environmental chamber has been constructed and established in accordance to our needs and focus in research, as we have presented and discussed above. However, there are some general and specific limitations that have to be considered.

Although this chamber is well suited for looking at the development of external structures and how they change in time, many changes, especially with respect to pathophysiology, take place internally and would not be seen with this imaging setup.

\section{Imaging of the Chick Heart Development}

The first phase of the cardiac looping process, which is characterized by the transformation of the straight heart tube into a c-shaped loop whose greater curvature points toward the right (Männer, 2000, 2004), cannot be visualized in ovo or SC. Starting at HH-stage 14 (50-55 h of incubation) the chick embryo lies on the yolk sac such that right-sided cardiovascular structures (delineated by red blood cells) become accessible for visual inspection through the transparent embryonic tissue. Thus, during the cardiac looping phases that are covered by our present study, the embryo is visualized only from the right lateral view, as shown in Figure 3. Left-sided structures are not visualized at any 
time and therefore cannot be assessed during the structural formation of the cardiovascular system, which takes approximately 9 to 10 days after incubation. Additionally, thickening of the initially thin and transparent thoracic wall and growth of the limb buds and "extra-embryonic" membranes prevent full visualization of the developing heart beyond day 4 (HH-stage 25).

\section{Adaptation of Our System for Other Animal Models}

Imaging studies with this system in zebrafish or Xenopus will require sedation of the organisms so that movement is minimized. Xenopus researchers interested in looking at very early morphogenesis could conduct their studies without sedation of the embryo. Studies in mouse will be demanding for various reasons: The mouse embryo develops in utero and to take the embryo out of the mom and culture is not easy and very limited in its time frame (the embryos are likely to survive only about $48 \mathrm{~h}$ and must be taken at a certain window in development; Fujinaga, 2000). Thus, imaging mouse embryos with this system will only be feasible for short time periods.

\section{Time-lapse Studies over Long Periods}

We have demonstrated the convenience of our system for image acquisition and have shown that hourly image samplings are possible without significant interruption of the temperature and humidity control, as the recordings are conducted within a time frame of a minute at maximum. Although the need to open the chamber for embryo positioning and to remove the Petri dish lid prior to recording is not of much concern in our hands, this could limit the utility of the system in terms of true time-lapse analysis over long periods for other purposes and applications. With regard to embryonic movement, one can imagine ever more expensive and sophisticated mechanical solutions to embryonic positioning in the imaging field and postprocessing imaging software that can help limit these issues without opening the chamber door and moving the embryo by hand. New generations of systems have to be developed in the future to address and to overcome these limitations.

\section{ACKNOWLEDGMENTS}

This work was supported by a research grant of the Braukmann-Wittenberg Foundation at Hannover Medical School and a research award endowed by HERZKIND e.V. (Maximilian-Forschungs-Förderpreis 2004) to T.M. Yelbuz. We thank Petra Wübbolt-Lehmann, Hannover Medical School, for her technical assistance in embryo preparations for shell-less culture.

\section{REFERENCES}

Borg, T.K., Stewart, J.A., Jr. \& Sutton, M.A. (2005). Imaging the cardiovascular system: Seeing is believing. Microsc Microanal 11, 189-199.

Burggren, W. \& Warburton, S. (2005). Comparative developmental physiology: An interdisciplinary convergence. Annu Rev Physiol 67, 203-223.

Conway, S.J., Godt, R.E., Hatcher, C.J., Leatherbury, L., Zolotouchnikov, V.V., Brotto, M.A., Copp, A.J., Kirby, M.L. \& Creazzo, T.L. (1997). Neural crest is involved in development of abnormal myocardial function. J Mol Cell Cardiol 29, 2675-2685.

De la Cruz, M.V. \& Markwald, R.R. (1998). Living Morphogenesis of the Heart. Boston, MA: Birkhauser.

Efimov, I.R., Nikolski, V.P. \& Salama, G. (2004). Optical imaging of the heart. Circ Res 95, 21-33.

Fujinaga, M. (2000). In vitro culture of rodent embryos during the early postimplantation period. In Methods in Molecular Biology, Vol. 135: Developmental Biology Protocols, Vol. I, Tuan, R.S. \& Lo, C.W. (Eds.), pp. 53-76. Totowa, NJ: Humana Press, Inc.

Hamburger, V. \& Hamilton, H.L. (1951). A series of normal stages in the development of the chick embryo. J Morphol 88, 49-92.

Harvey, R.P. \& Rosenthal, N. (1999). Heart Development. London: Academic Press.

Keller, B.B. (1998). Embryonic cardiovascular function, coupling, and maturation: A species view. In Development of Cardiovascular Systems, Burggren, W.W. \& Keller, B.B. (Eds.), pp. 65-87. Cambridge, UK: Cambridge University Press.

Kolker, S.J., Tajchman, U. \& Weeks, D.L. (2000). Confocal imaging of early heart development in Xenopus laevis. Dev Biol 218, $64-73$.

Leatherbury, L., Gauldin, H.E., Waldo, K. \& Kirby, M.L. (1990). Microcinephotography of the developing heart in neural crest-ablated chick embryos. Circulation 81, 1047-1057.

Li, Y.X., Zdanowicz, M., Young, L., Kumiski, D., Leatherbury, L. \& Kirby, M.L. (2003). Cardiac neural crest in zebrafish embryos contributes to myocardial cell lineage and early heart function. Dev Dyn 226, 540-550.

MänNeR, J. (2000). Cardiac looping in the chick embryo: A morphological review with special reference to terminological and biomechanical aspects of the looping process. Anat Rec 259, 248-262.

MäNNER, J. (2004). On rotation, torsion, lateralization, and handedness of the embryonic heart loop: New insights from a simulation model for the heart loop of chick embryos. Anat Rec A Discov Mol Cell Evol Biol 278, 481-492.

Phoon, C.K. (2001). Circulatory physiology in the developing embryo. Curr Opin Pediatr 13, 456-464.

Soufan, A.T., Ruijter, J.M., van den Hoff, M.J., De Boer, P.A., Hagoort, J. \& Moorman, A.F. (2003). Three-dimensional reconstruction of gene expression patterns during cardiac development. Physiol Genomics 13, 187-195.

Tomanek, R.J. \& Runyan, R.B. (2001). Formation of the Heart and Its Regulation. Boston, MA: Birkhauser.

Tomita, H., Connuck, D.M., Leatherbury, L. \& Kirby, M.L. (1991). Relation of early hemodynamic changes to final cardiac 
phenotype and survival after neural crest ablation in chick embryos. Circulation 84, 1289-1295.

Tutarel, O., Norozi, K., Hornung, O., Orhan, G., WüвboltLehmann, P., Wessel, A. \& Yelbuz, T.M. (2005). Cardiac failure in the chick embryo resembles heart failure in humans. Circulation 112, e352-e353.

Ward, C., Stadt, H., Hutson, M. \& Kirby, M.L. (2005). Ablation of the secondary heart field leads to tetralogy of Fallot and pulmonary atresia. Dev Biol 284, 72-83.

Yelbuz, T.M., Сhoma, M.A., Thrane, L., Kirby, M.L. \& Izatt, J.A. (2002a). Optical coherence tomography: A new highresolution imaging technology to study cardiac development in chick embryos. Circulation 106, 2771-2774.

Yelbuz, T.M., Leatherbury, L., Wolfe, R.R., Loewy, R. \& Kirby, M.L. (2002b). Time-lapse study with high speed video camera in the early embryonic chick heart to visualize a time window of normal and abnormal heart development. Circulation 106, e44-e45.
Yelbuz, T.M., Waldo, K.L., Kumiski, D.H., Stadt, H.A., Wolfe, R.R., Leatherbury, L. \& Kirby, M.L. (2002c). Shortened outflow tract leads to altered cardiac looping after neural crest ablation. Circulation 106, 504-510.

Yelbuz, T.M., Waldo, K.L., Zhang, X., Zdanowicz, M., Parker, J., Creazzo, T.L., Johnson, G.A. \& Kirby, M.L. (2003a). Myocardial volume and organization are changed by failure of addition of secondary heart field myocardium to the cardiac outflow tract. Dev Dyn 228, 152-160.

Yelbuz, T.M., Wessel, A. \& Kirby, M.L. (2004). [Studies on morphogenesis and visualization of the early embryonic heart with regard to the development of conotruncal heart defects]. $Z$ Kardiol 93, 583-594.

Yelbuz, T.M., Zhang, X., Choma, M.A., Stadt, H.A., Zdanowicz, M., Johnson, G.A. \& Kirby, M.L. (2003b). Approaching cardiac development in three dimensions by magnetic resonance microscopy. Circulation 108, e154-e155. 